Supporting Information

\title{
Phase Evolution, Polymorphism and Catalytic Activity of Nickel Dichalcogenide Nanocrystals
}

Marquix A. S. Adamson, ${ }^{1}$ Philip Yox,,${ }^{1,2}$ Thomas Hernandez, ${ }^{1}$ Fei Wang, ${ }^{3}$ Javier Vela ${ }^{1,2, *}$

${ }^{1}$ Department of Chemistry, Iowa State University, Ames, Iowa 50011, United States, ${ }^{2}$ Ames Laboratory, Ames, Iowa 50011, United States, and ${ }^{3}$ Department of Chemistry, Missouri State University, Springfield, Missouri 65897, United States.

*email: vela@iastate.edu, phone: 515-294-5536 


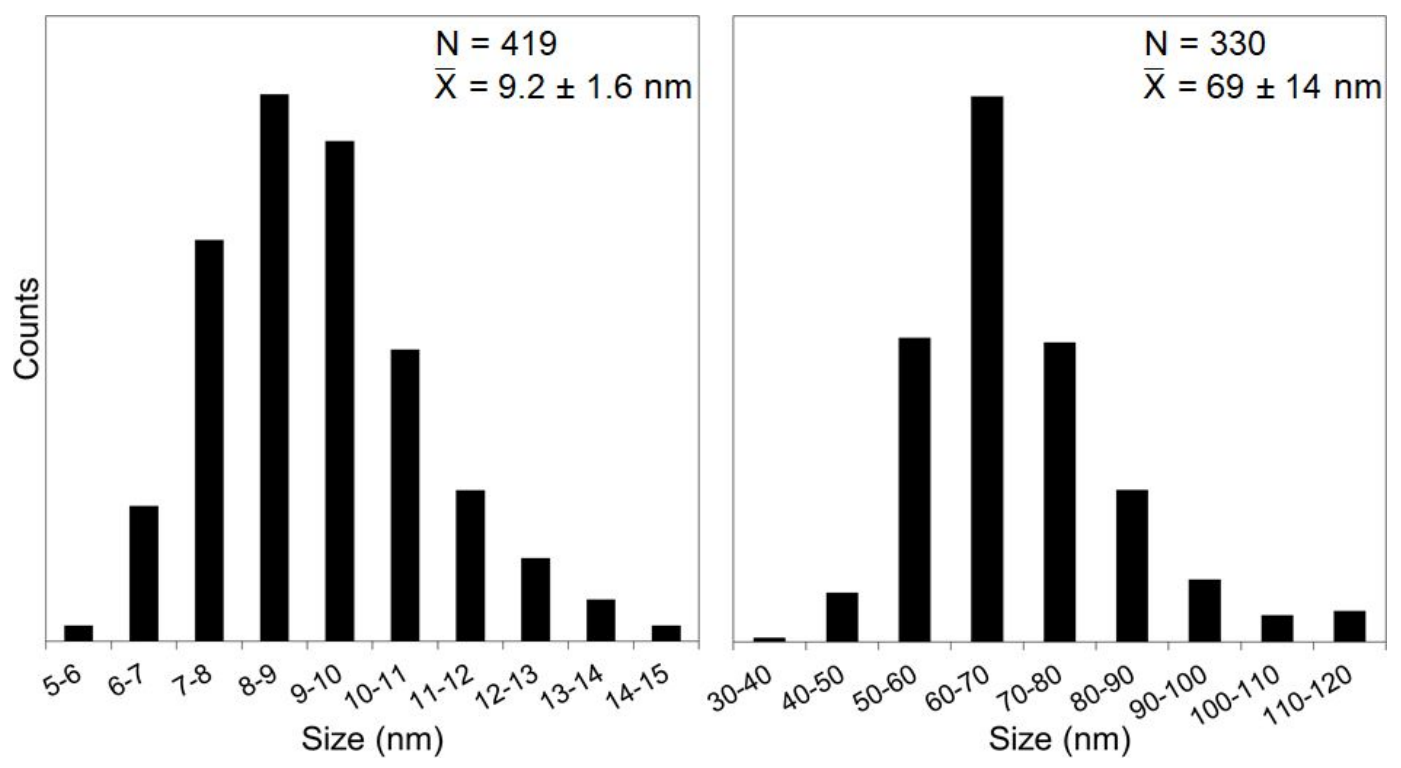

Figure S1. Size histograms of individual (left) and aggregated (right) $\mathrm{NiS}_{2}$ nanocrystals synthesized from $\mathrm{Ni}$ (acetate) $)_{2} \cdot 4 \mathrm{H}_{2} \mathrm{O}(0.1 \mathrm{M})$ and $\mathrm{S}(1 \mathrm{M})$ in oleylamine at $240{ }^{\circ} \mathrm{C}$ for $10 \mathrm{~min}$ (see Methods).

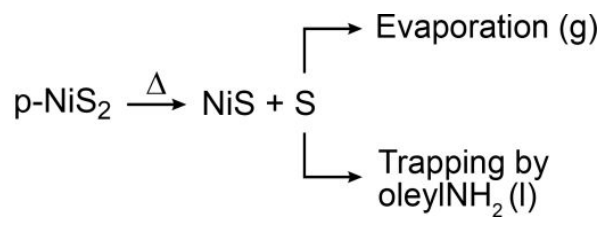

Scheme S1. Decomposition of $\mathrm{NiS}_{2}$ into $\alpha-\mathrm{NiS}$ and S. 
Table S1. Thermodynamic data used in Table S2. ${ }^{\text {s1 }}$

\begin{tabular}{cccc}
\hline Compound & Note $(\mathrm{s})$ & $\Delta \mathrm{H}_{\mathrm{f}}^{\circ}(\mathrm{kJ} / \mathrm{mol})$ & $\mathrm{S}_{\mathrm{f}}(\mathrm{J} / \mathrm{mol} \cdot \mathrm{K})$ \\
\hline $\mathrm{NiS}_{2}$ & Solid & -131.38 & 71.97 \\
$\mathrm{NiS}$ & Solid & -87.86 & 52.99 \\
$\mathrm{~S}$ & Solid & 0 & 32.05 \\
\hline
\end{tabular}

Table S2. Thermodynamics of $\mathrm{NiS}_{2}$ decomposition.

\begin{tabular}{ccc}
\hline Equation & $\Delta \mathrm{H}_{\mathrm{r}}^{\circ}(\mathrm{kJ} / \mathrm{mol})$ & $\mathrm{S}_{\mathrm{r}}^{\circ}(\mathrm{J} / \mathrm{mol} \cdot \mathrm{K})$ \\
\hline$(\mathrm{NiS}+\mathrm{S})-\mathrm{NiS}_{2}$ & 43.52 & 13.07 \\
\hline
\end{tabular}

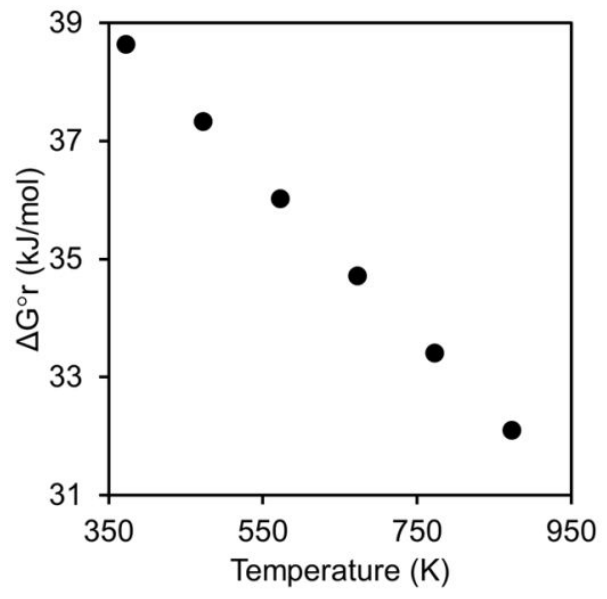

Figure S2. Calculated $\Delta \mathrm{G}^{\circ}$ for the decomposition of $\mathrm{NiS}_{2}$ as a function of temperature. 


$$
\overbrace{\mathrm{NH}_{2}}+\mathrm{S}_{\mathrm{n}}{ }^{2-} \stackrel{\Delta}{\longrightarrow} \overbrace{\mathrm{NH}_{2}}^{\mathrm{S}}+\mathrm{H}_{2} \mathrm{~S}(\mathrm{~g})+\mathrm{S}_{\mathrm{n}-2}{ }^{2-}
$$

Scheme S2. Trapping of sulfur by oleylamine.
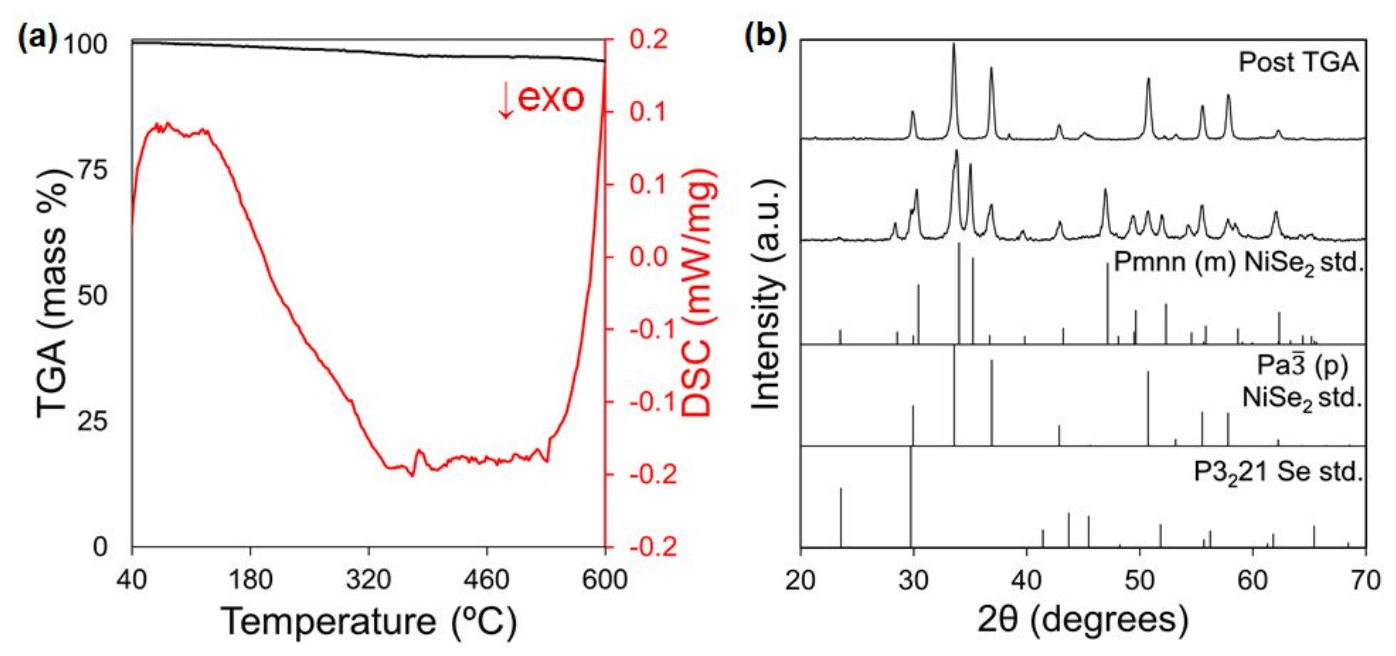

Figure S3. (a) Thermal analysis (TGA-DSC) and powder XRD (b) of a mixture of a ca. 1:1 marcasiteand pyrite-NiSe $\mathrm{N}_{2}$ upon annealing to $600{ }^{\circ} \mathrm{C}\left(10{ }^{\circ} \mathrm{C} / \mathrm{min}\right)$. 


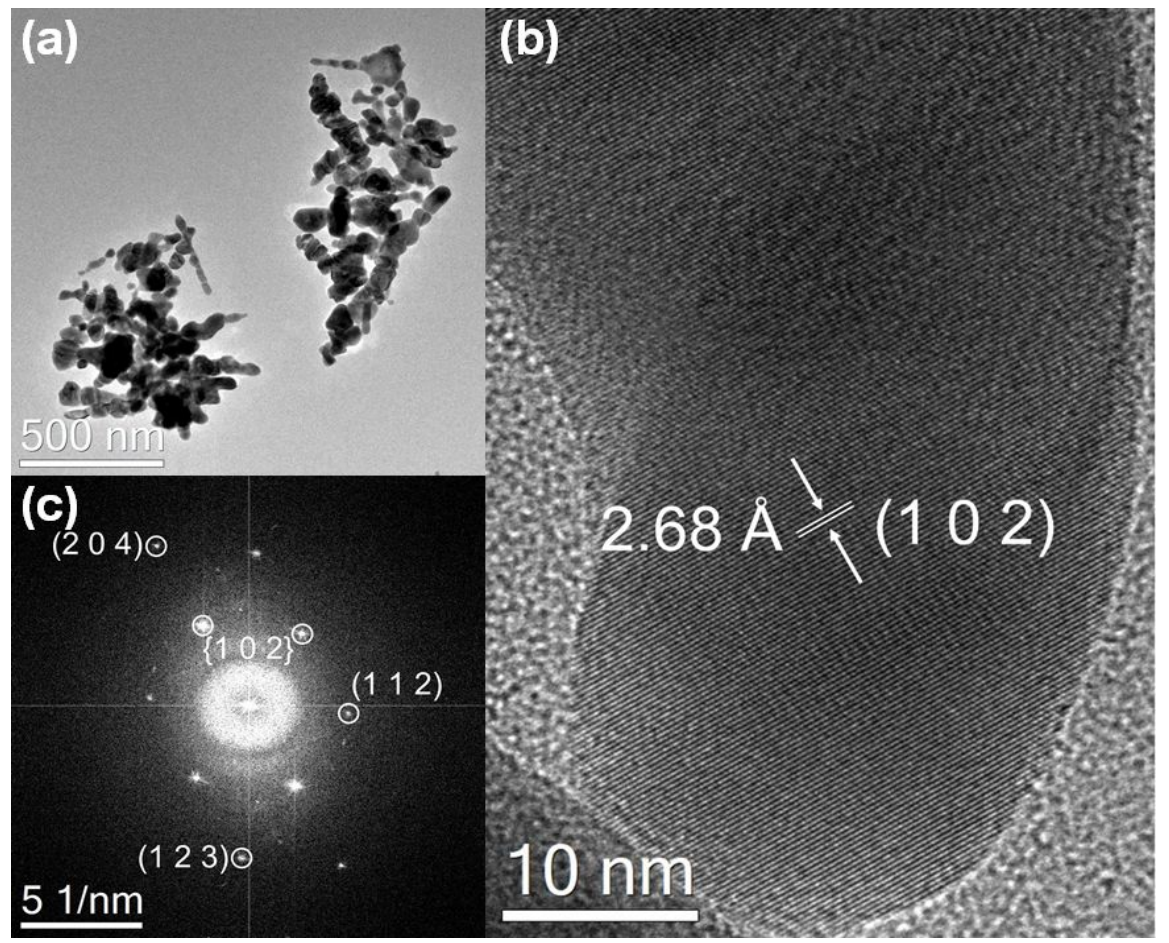

Figure S4. Representative TEM (a), HR TEM (b), and FFT (b) of $\mathrm{NiSe}_{2}$ nanocrystals synthesized from $\mathrm{Ni}$ (acetate) $)_{2} \cdot 4 \mathrm{H}_{2} \mathrm{O}(0.1 \mathrm{M})$ and $\mathrm{Se}(0.5 \mathrm{M})$ in oleylamine at $260^{\circ} \mathrm{C}$ for $60 \mathrm{~min}$ (see Methods). 

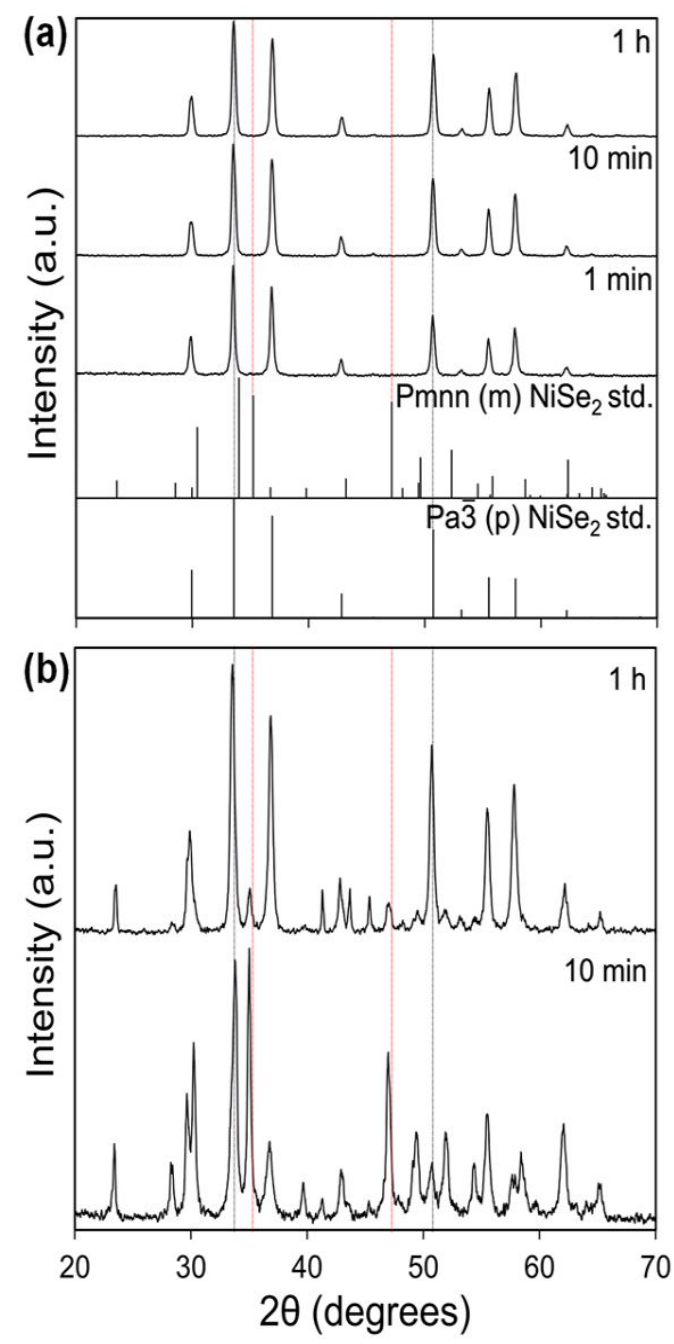

Figure S5. Powder XRD patterns of nickel diselenide nanocrystals synthesized in oleylamine at different reaction times at $260{ }^{\circ} \mathrm{C}$ (a) or $200{ }^{\circ} \mathrm{C}$ (b) $(0.1 \mathrm{M} \mathrm{Ni} \text { (stearate) })_{2}, 0.5 \mathrm{M} \mathrm{Se}$, see Methods). 

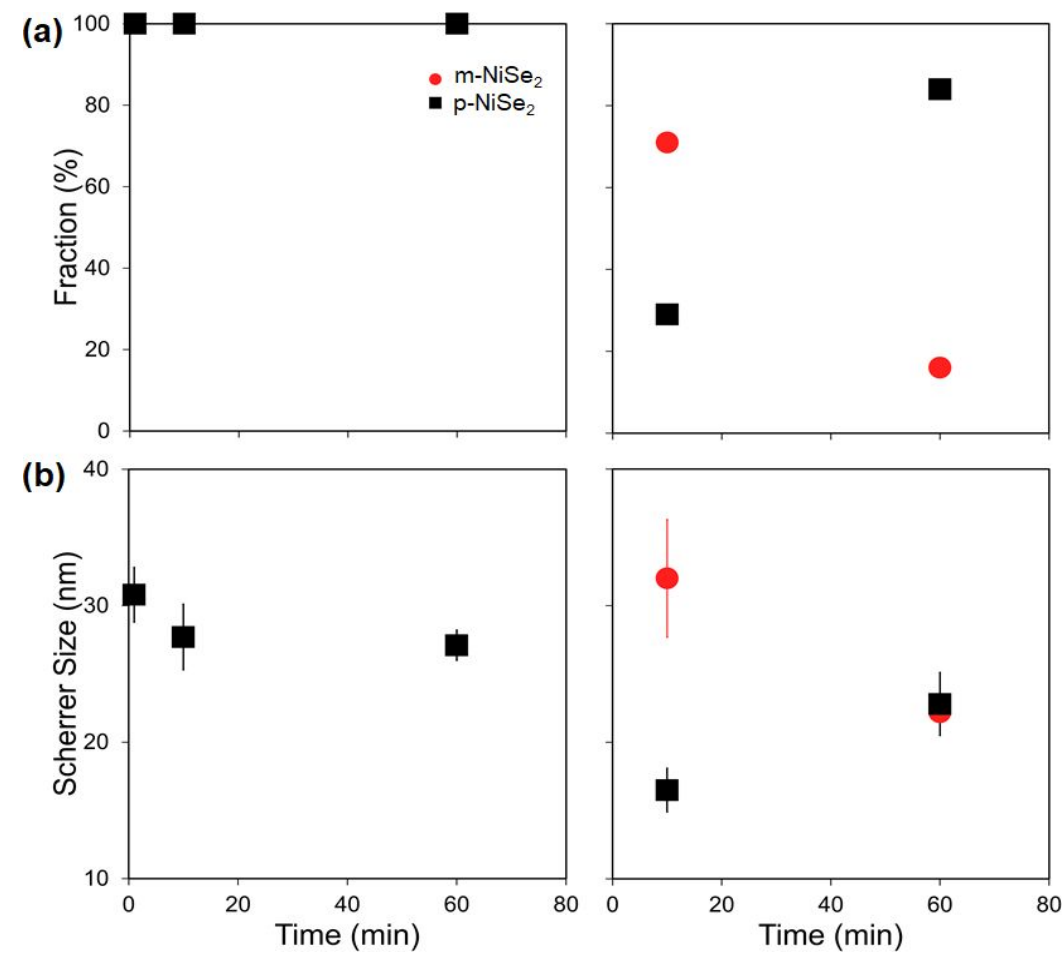

Figure S6. Percentage of crystalline products (a) and average Scherrer size (b) for nickel diselenide nanocrystals synthesized in oleylamine under different conditions at $260^{\circ} \mathrm{C}$ (left) or $200^{\circ} \mathrm{C}$ (right) $(0.1 \mathrm{M}$ $\mathrm{Ni}(\text { stearate) })_{2}, 0.5 \mathrm{M}$ Se, see Methods). 


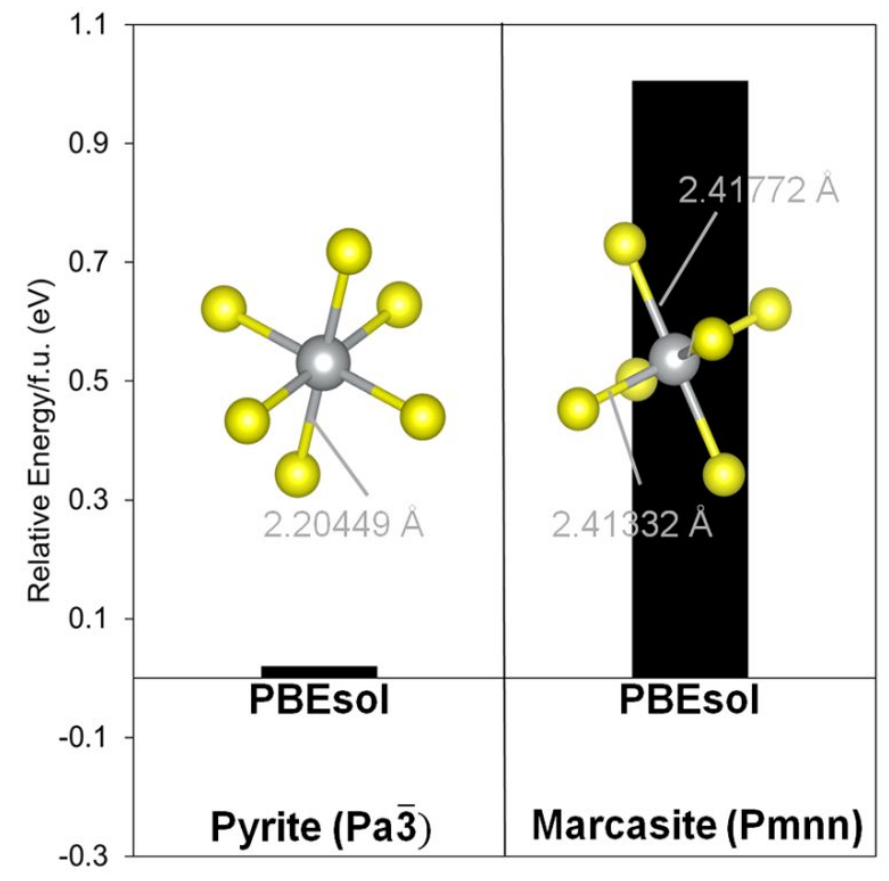

Figure S7. Total energies and Ni-Se bond lengths calculated using PBEsol when using a $20 \times 20 \times 20 \mathrm{k}-$ point mesh, and optimizing cell volume, cell shape, and atomic positions.

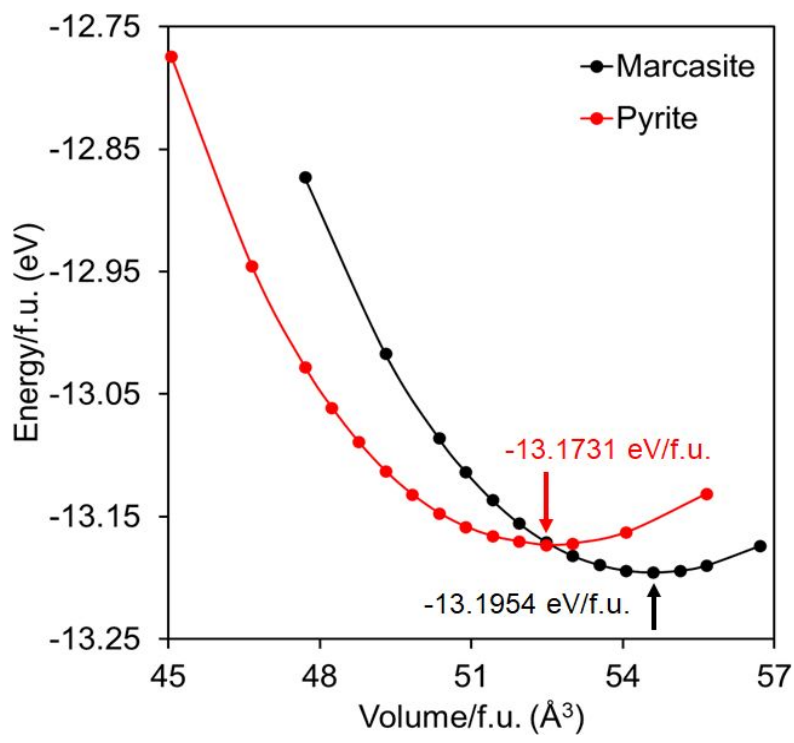

Figure S8. PBE-based volume scans of $\mathrm{p}-\mathrm{NiSe}_{2}$ and $\mathrm{m}-\mathrm{NiSe}_{2}$ without Birch-Murnaghan equation of state fitting. 


\begin{tabular}{|c|c|c|c|c|}
\hline \multicolumn{5}{|c|}{ Table S3. Assessing $\mathrm{NiSe}_{2}$ polymorphism from calculations. ${ }^{a}$} \\
\hline $\begin{array}{c}\text { Space } \\
\text { Group }\end{array}$ & Functional & $\begin{array}{c}\text { Energy/f.u. } \\
(\mathrm{eV})\end{array}$ & $\begin{array}{c}\text { Ni-Se bond } \\
\text { distances } \\
(\AA)\end{array}$ & $\begin{array}{c}\text { Volume/f.u. } \\
\left(\AA^{3}\right)\end{array}$ \\
\hline \multirow{4}{*}{$P a \overline{3}$} & PBE & -13.1757 & 2.47 & 52.42 \\
\cline { 2 - 5 } & PBEsol $^{b}$ & -14.7612 & 2.41 & 49.54 \\
\cline { 2 - 5 } & LDA $^{*}$ & -15.8968 & 2.39 & 48.25 \\
\hline \multirow{3}{*}{ Pmnn } & PBE & -13.1985 & $2.39,2.40$ & 54.52 \\
\cline { 2 - 5 } & PBEsol $^{b}$ & -14.7350 & $2.41,2.42$ & 51.83 \\
\cline { 2 - 5 } & LDA & -15.8639 & $2.45,2.48$ & 50.54 \\
\hline
\end{tabular}

${ }^{a}$ Total energy calculations were performed using a $20 \times 20 \times 20 \mathrm{k}-$ point mesh optimizing cell volume, cell shape, and atomic position. ${ }^{b}$ Values are from minima of volume scans using PBEsol.

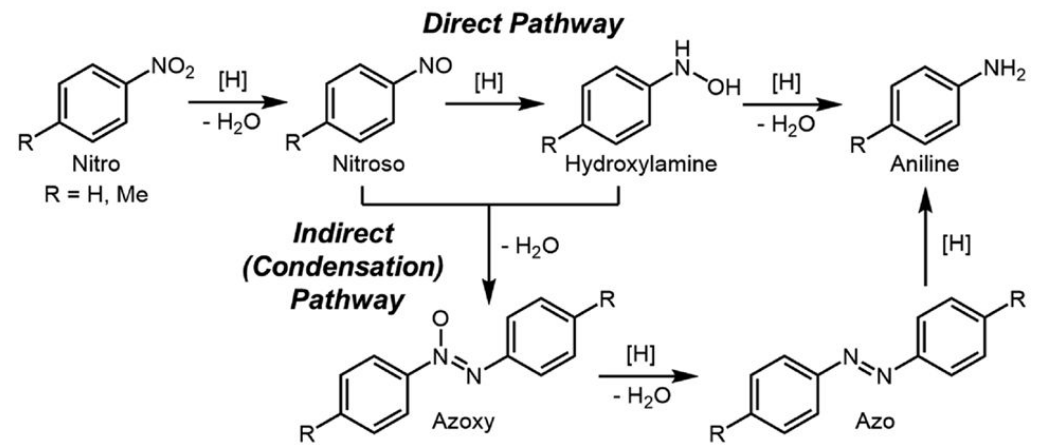

Scheme S3. Nitroarene reduction pathways. Reprinted with permission from Daniels, C. L.; Liu, D.-J.; Adamson, M. A. S.; Knobeloch, M.; Vela, J. "Azo(xy) vs Aniline Selectivity in Catalytic Nitroarene Reduction by Intermetallics: Experiments and Simulations." J. Phys. Chem. C 2021, 125, 24440-24450. Copyright 2021 American Chemical Society. ${ }^{\text {S2 }}$

S1 NIST Standard Reference Database 13. U.S. Department of Commerce https://janaf.nist.gov/ (accessed 2021-1012).

S2 Daniels, C. L.; Liu, D.-J.; Adamson, M. A. S.; Knobeloch, M.; Vela, J. "Azo(xy) vs Aniline Selectivity in Catalytic Nitroarene Reduction by Intermetallics: Experiments and Simulations." J. Phys. Chem. C 2021, 125, 24440-24450. 